\title{
Augmenting the randomized controlled trial with real-world data to aid clinical decision making in metastatic renal cell carcinoma: a systematic review and meta-analysis
}

\author{
Michael Moran*,1, Dana Nickens ${ }^{2}$, Katherine Adcock ${ }^{3}$, Meg Bennetts ${ }^{4}$, Natalie Charnley ${ }^{5}$ \& \\ Kate Fife $^{6}$ \\ ${ }^{1}$ Pfizer GmbH, Pfizer Oncology, Berlin 10785, Germany \\ 2Pfizer Global, La Jolla, CA 90630, USA \\ ${ }^{3}$ Pfizer Ltd, Walton Oaks, UK \\ ${ }^{4}$ Pfizer R\&D UK Ltd, Sandwich, UK \\ ${ }^{5}$ Lancashire Teaching Hospitals, Cancer Services, Lancashire, UK \\ ${ }^{6}$ Cambridge University Hospital, Cancer Services, Cambridge, UK \\ *Author for correspondence: Tel.: +44 758414 5766; michael.m.moran@pfizer.com
}

\begin{abstract}
Aim: To evaluate how efficacy outcomes from real-world data (RWD) can support those from randomized controlled trials (RCTs), in the context of first-line tyrosine kinase inhibitor treatment of metastatic renal cell carcinoma. Patients \& methods: PubMed, Ovid, MEDLINE and EMBASE were searched for RCTs and RWD studies with $\geq 50$ adult patients per arm published in 2000-2017. Outcome measures were median progression-free survival, median overall survival and objective response rate. Results: A total of 13 RCTs and 22 RWD studies met eligibility criteria; 31, 28 and 25 studies, respectively, reported median progression-free survival, median overall survival and objective response rate. Summary outcome measures were similar in RWD and RCTs. Conclusion: RWD validates efficacy-based outcomes from RCTs and may provide supportive evidence to inform clinical decisions.
\end{abstract}

\section{Lay abstract:}

- Clinical trials are an established way of gathering evidence to show that new drugs are effective.

- There are concerns that clinical trials do not fully represent those patients seen in everyday clinical practice.

- 'Real-world' studies look at the effect of drugs under normal conditions (everyday clinical practice).

- This study compared the results from 13 clinical trials and 22 real-world studies for a group of drugs called tyrosine kinase inhibitors in patients with metastatic renal cell carcinoma, a type of kidney cancer that has spread to other parts of the body.

- Overall, results from real-world studies were similar to those from clinical trials.

- This suggests that results from real-world studies may help doctors decide on the most effective treatment.

First draft submitted: 18 July 2019; Accepted for publication: 24 September 2019; Published online: 18 October 2019

Keywords: clinical trials $\bullet$ real-world data $\bullet$ renal cell carcinoma $\bullet$ tyrosine kinase inhibitor

Randomized controlled trials (RCTs) are the gold standard for obtaining evidence for the regulatory approval of new medical interventions. These trials are designed to produce data of high internal validity, which is to minimize the potential for bias and confounding [1]. This is achieved through stringent eligibility criteria and intensive monitoring to ensure a strictly defined study protocol is followed [2]. Thus, RCTs provide robust evidence that the medical intervention in question genuinely has an effect in the study population. Evidence from RCTs is used to inform healthcare policies and clinical decision making; however, clinicians have long been concerned that the

Future 8 Medicine 
generalizability (external validity) of RCTs is often insufficient [3,4]. Low external validity can lead to an over-reliance on clinical judgement to extrapolate evidence from RCTs, which may introduce inconsistent practice and quality of care [5].

Real-world data (RWD), which can be broadly defined as data from sources outside of the conventional controlled clinical research setting, are becoming increasingly valuable for healthcare professionals [6]. The increased use of electronic healthcare records, advances in data science technology and provisions for safe storage of clinical data present an opportunity to utilize RWD in healthcare and clinical decision making [7]. Although RWD have limited internal validity, compared with RCTs, these data can provide complementary evidence for the effectiveness and safety of medical interventions in larger, heterogeneous populations of patients, therefore, expanding the evidence base upon which clinical decisions are made $[8,9]$.

The potential of RWD to supplement evidence from RCTs may be especially relevant in oncology where it is estimated that $<5 \%$ of patients with cancer are enrolled in RCTs [10,11]. Patient participation in cancer RCTs has traditionally been poor, especially for elderly patients and certain ethnic groups [10]. First-line treatment RCTs and randomization may be perceived negatively by patients, with one study reporting that $>80 \%$ of patients with cancer would be unwilling to participate in RCTs [11]. Premature closure of oncology trials due to insufficient accrual is common, which increases costs and hinders medical advances [12]. These issues have added to concerns regarding the generalizability of RCTs. In August 2018, the American Society of Clinical Oncology and nonprofit organization Friends of Cancer Research submitted draft guidance documents to the US FDA to broaden eligibility criteria of cancer RCTs to improve their generalizability [13].

Each year, there are an estimated 400,000 newly diagnosed cases of kidney cancer worldwide [14]. Renal cell carcinoma (RCC) accounts for approximately $90 \%$ of these cases [15], with $>20 \%$ of patients presenting with metastatic RCC (mRCC) [16]. Partial or radical nephrectomy is usually the first treatment for primary RCC [15,17,18]; however, depending on risk factors and tumor stage, at least $20 \%$ of patients with localized RCC will develop recurrences within 5 years' post nephrectomy [16]. The estimated 5-year survival rate for patients with locoregional disease is $53 \%$, which falls to $8 \%$ for patients with metastatic disease [19].

Targeted therapy, including tyrosine kinase inhibitors (TKIs), revolutionized the treatment of mRCC. Since their development, several TKIs, including sunitinib and pazopanib, have been available and recommended as a first-line systemic treatment for favorable risk patients with mRCC $[15,17,18]$. The rapid development of new treatments for mRCC, including combination therapies, has added a layer of complexity to treatment management. First-line therapies need to be strategically chosen to optimize sequential therapy lines in patients with mRCC [20].

The aim of this systematic review and meta-analysis was to address how efficacy outcomes from real-world studies can support those from RCTs, in the context of TKI therapies for first-line management of mRCC.

\section{Materials \& methods}

\section{Search strategy}

This systematic review and meta-analysis was conducted and reported based on the Preferred Reporting Items for Systematic Reviews and Meta-Analyses (PRISMA) guidelines [21]. Methods of the analysis, outcome measures and inclusion criteria were prespecified in a protocol. No changes were subsequently made to the protocol.

A systematic literature review of first-line TKI treatment in patients with mRCC published from 2000 to 2017 was conducted using a comprehensive search of PubMed, Ovid, MEDLINE and EMBASE according to the Population, Intervention, Comparator, Outcomes Study Designs (PICOS) framework (Table 1). The literature search was initiated and completed in July 2018. Only English language publications were included in the search, which has been reported to have no impact on systemic bias [22]. The following terms were used to search all databases: 'kidney', 'renal', 'carcinoma*', 'cancer*', 'neoplasm*', 'tumo?r', 'malignan*', 'oncolog*', 'metastatic*', 'metastasis*', in combination with the terms 'first line', '1st line', 'initial*', 'treatment*', 'therap*' and 'intervention*'. Eligible studies were RWD studies and RCTs that reported outcomes for previously untreated adults diagnosed with mRCC and had a minimum cohort of 50 patients in each treatment group receiving a TKI as first-line therapy (Table 1). Patients who did not have a clearly defined diagnosis of advanced or metastatic renal cell carcinoma were excluded. The interventions assessed were TKIs as a single agent in the first-line setting in RWD studies (RWD-TKI), TKIs as a single agent in the first-line setting in RCTs (RCT-TKI) and matching control non-TKI treatments in RCTs (RCT-control), which were included to support the assessment of between-study heterogeneity. If more than one publication was found for the same clinical study, the most recent analysis was included and duplicates excluded. 


\begin{tabular}{|c|c|c|}
\hline PICOS element & Inclusion & Exclusion \\
\hline Patient population & $\begin{array}{l}\text { Men and women; } \\
\text { aged }>18 \text { years; } \\
\text { mRCC population; } \\
\text { first-line treatment; } \\
\text { January } 2000 \text {-December } 2017 ; \\
\text { cohorts } \geq 50 \text { patients }\end{array}$ & $\begin{array}{l}\text { Pediatric patients; } \\
\text { mixed-disease population; } \\
\text { cohorts }<50 \text { patients }\end{array}$ \\
\hline Intervention & $\begin{array}{l}\text { Medical treatment for mRCC } \\
\text { TKI }\end{array}$ & $\begin{array}{l}\text { Non-TKI treatment; } \\
\text { prior therapy }\end{array}$ \\
\hline Comparator & $\begin{array}{l}\text { Research study outcomes from RCTs and retrospective } \\
\text { studies }\end{array}$ & \\
\hline Outcomes & Efficacy: PFS, OS, ORR & Non-efficacy based \\
\hline Study design & $\begin{array}{l}\text { Phase II and III RCTs; } \\
\text { RWD; } \\
\text { health records; } \\
\text { cohort studies; } \\
\text { case-controlled studies; } \\
\text { cross-sectional studies }\end{array}$ & $\begin{array}{l}\text { Phase I RCT; } \\
\text { case reports; } \\
\text { animal and preclinical trials; } \\
\text { opinion papers and letters; } \\
\text { systematic reviews and meta-analyses }\end{array}$ \\
\hline
\end{tabular}

\section{Outcome measures}

Outcomes recorded as aggregate data were median progression-free survival (mPFS), median overall survival (mOS) and objective response rate (ORR). Covariates extracted from each study were age and duration of therapy.

\section{Study selection \& data extraction}

Two investigators (M Moran and K Adcock) conducted the literature search independently. Disagreements were resolved through discussion and consensus. Relevant data were identified by K Adcock and extracted by D Nickens into a format suitable for analysis. Data were independently quality checked against source material by M Bennetts.

\section{Risk of bias}

Publication bias was assessed by investigator review of funnel plots for each end point.

Potential bias over time was investigated via a cumulative time forest plot that calculated a series of meta-analysis estimates in chronological order of study publication year for each end point (mPFS, mOS and ORR) and study type (RCT vs RWD).

\section{Analyses}

A linear mixed-effects model was fitted to log-transformed time-to-event end point data (mPFS, mOS) with effects to reflect: RWD-TKI (categorical covariate [= 1 or 0 otherwise] for TKI treatment groups from RWD), RCT-TKI (categorical covariate [ 1 or 0 otherwise] for TKI treatment groups from RCTs), and RCT-control (categorical covariate [ $=1$ or 0 otherwise] for non-TKI treatment groups from RCTs), a between-study random effect ( $\eta_{i} \sim$ $\left.N\left[0, \tau^{2}\right]\right)$ and a residual random error term $\left(\epsilon_{i j} \sim N\left[0, \sigma^{2} / N_{i j}\right]\right)$, where $\mathrm{i}=$ study, $\mathrm{j}=$ treatment arm and $\theta=$ average time-to-event (months) for the specific treatment group:

$$
\log \left(T T E_{i j}\right)=\theta_{1} * \mathrm{RCT}-\text { control }+\theta_{2} * \mathrm{RCT}--\mathrm{TKI}+\theta_{2} * \mathrm{RWD}--\mathrm{TKI}+\eta_{i}+\varepsilon_{i j}
$$

A similar model was used to analyze ORR using a logit transformation and assuming binomial distribution $\left(O R R_{i j} \sim\right.$ binomial $\left.\left[N_{i j}, p_{i j}\right]\right)$, where $\mathrm{N}_{\mathrm{ij}}=$ number of patients per study per treatment arm, $\mathrm{p}_{\mathrm{ij}}=$ probability of objective response in the study and treatment arm:

$$
\operatorname{logit}\left(N_{i j}\right)=\theta_{1} * \mathrm{RCT}-\mathrm{control}+\theta_{2} * R C T--T K I+\theta_{2} * \mathrm{RWD}--\mathrm{TKI}+\eta_{i}
$$

All statistical tests were conducted using a significance level of 0.05 . Study heterogeneity $\left(\tau^{2}\right)$ was estimated via inclusion of a between-study random effect $\left(\eta_{i}\right)$ in each end point model. Covariates were assessed by visual 


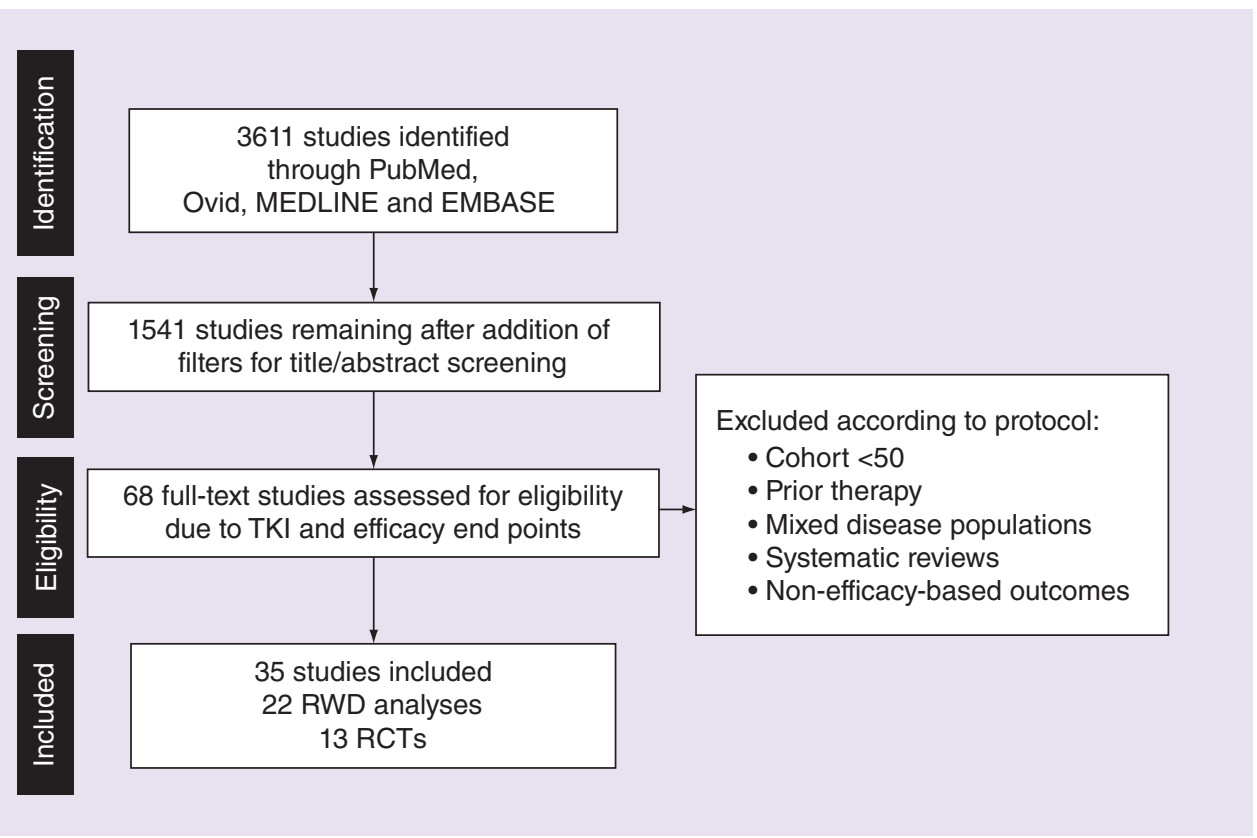

Figure 1. PRISMA flow diagram of the literature search process.

PRISMA: Preferred reporting items for systematic reviews and meta-analyses; RCT: Randomized controlled trial; RWD: Real-world data; TKI: Tyrosine kinase inhibitor.

inspection of observed data scatter plots. A prespecified subgroup analysis of first-line sunitinib-treated patients was also performed, which aimed to provide an estimate of sunitinib efficacy by combining RWD and data from RCTs [23].

\section{Results}

Search results

A total of 3611 studies were identified, of which 35 studies (13 RCT [24-37], 22 RWD [38-59]; Supplementary Tables $1 \& 2$ ) met the inclusion criteria and were included in analyses (Figure 1). Of the 35 included studies, 31, 28 and 25 reported mPFS, mOS and ORR, respectively. The number of studies, treatment groups, patients and type of TKI per efficacy end point are summarized in Table 2. Overall, RCT studies included a broader range of TKIs, whereas RWD were limited to three TKIs (pazopanib, sorafenib and sunitinib). Sunitinib was the most commonly investigated TKI in this analysis for both RCTs and RWD.

There was no strong evidence of publication bias among studies reporting mPFS, mOS or ORR (Supplementary Figure 1A-C); however, there was evidence of a potential time bias for RCTs. The RCTs included in this analysis span a wider set of years than the RWD studies, which were predominantly clustered in more recent years (20142017) (Table 3). The cumulative estimate of mPFS in RCTs increased from 3.5 months in 2008 to 8.4 months in 2017 (Supplementary Figure 2A). There was little evidence of a potential time bias in RWD; the cumulative estimate of mPFS increased from 8.9 months in 2010 to 9.6 months in 2017 (Supplementary Figure 2B). A similar pattern was observed for mOS (Supplementary Figure 3) and ORR (Supplementary Figure 4).

\section{Efficacy meta-analysis}

Summary measures representing 8493, 8899 and 7711 patients were analyzed for mPFS, mOS and ORR, respectively.

Observed data for mPFS, mOS and ORR are shown in Figures 2, $3 \&$ 4, respectively. RCTs reported a wider range of mPFS values compared with RWD studies (Figure 2). Overall, mPFS was numerically longer in RWD versus RCT (9.4 [95\% CI: 8.1-11.0] vs 8.6 [95\% CI: 7.3-10.3] months), but the difference did not reach statistical significance $(\mathrm{p}=0.4505)$ (Figure $5 \mathrm{~A})$. The overall estimate for $\mathrm{mOS}$ was numerically longer in RCTs versus RWD (26.2 [95\% CI: 19.6-35.0] vs 20.8 [95\% CI: 17.5-24.8] months); however, the difference was not 


\begin{tabular}{|c|c|c|c|c|c|}
\hline End point & Group & Number of studies & $\begin{array}{l}\text { Cumulative treatment } \\
\text { arms }\end{array}$ & Patients, $\mathbf{n}^{\dagger}$ & 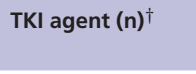 \\
\hline \multirow[t]{3}{*}{ mPFS } & RWD-TKI & 18 & 20 & 4771 & $\begin{array}{l}\text { Pazopanib (636) } \\
\text { Sorafenib (1037) } \\
\text { Sunitinib (3098) }\end{array}$ \\
\hline & RCT-TKI & 13 & 18 & 3427 & $\begin{array}{l}\text { Axitinib (248) } \\
\text { Cabozantinib (79) } \\
\text { Lapatinib (209) } \\
\text { Pazopanib (557) } \\
\text { Sorafenib (436) } \\
\text { Sunitinib (1717) } \\
\text { Tivozanib (181) }\end{array}$ \\
\hline & RCT-control & 8 & 8 & 1295 & NA \\
\hline \multirow[t]{2}{*}{$\mathrm{mOS}$} & RWD-TKI & 20 & 23 & 5622 & $\begin{array}{l}\text { Pazopanib (729) } \\
\text { Sorafenib (921) } \\
\text { Sunitinib (3972) }\end{array}$ \\
\hline & RCT-control & 6 & 6 & 965 & NA \\
\hline \multirow[t]{3}{*}{ ORR } & RWD-TKI & 15 & 17 & 3925 & $\begin{array}{l}\text { Pazopanib (406) } \\
\text { Sorafenib (825) } \\
\text { Sunitinib (2694) }\end{array}$ \\
\hline & RCT-TKI & 10 & 14 & 2820 & $\begin{array}{l}\text { Axitinib (248) } \\
\text { Cabozantinib (76) } \\
\text { Lapatinib (209) } \\
\text { Pazopanib (551) } \\
\text { Sorafenib (247) } \\
\text { Sunitinib (1489) }\end{array}$ \\
\hline & RCT-control & 6 & 6 & 966 & NA \\
\hline
\end{tabular}

† Patient numbers as represented by the aggregate data.

mOS: Median overall survival; mPFS: Median progression-free survival; NA: Not applicable; ORR: Objective response rate; RCT: Randomized controlled trial; RCT-control: Matching control, non-TKI treatments in RCT; RWD: Real-world data; TKI: Tyrosine kinase inhibitor.

Table 3. Number of studies by publication year and study type for median progression-free survival.

\begin{tabular}{|lll|}
\hline Publication year & RCT & RWD \\
\hline 2008 & 1 & 0 \\
\hline 2009 & 2 & 0 \\
\hline 2010 & 0 & 1 \\
\hline 2011 & 1 & 0 \\
\hline 2012 & 1 & 0 \\
\hline 2013 & 3 & 0 \\
\hline 2014 & 1 & 3 \\
\hline 2015 & 0 & 6 \\
\hline 2016 & 3 & 5 \\
\hline 2017 & 1 & 3 \\
\hline RCT: & & \\
\hline
\end{tabular}

RCT: Randomized controlled trial; RWD: Real-world data.

statistically significant $(\mathrm{p}=0.1643)$ (Figure $5 \mathrm{~B})$. Overall, the ORR was similar for RWD and RCTs $(26.5 \%$ [95\% CI: 19.6-34.8] vs 25.0\% [95\% CI: 17.1-35.0]; $\mathrm{p}=0.7990$ ) (Figure 5C).

Efficacy data from RWD studies of elderly patients (mPFS: 8.8-11.0 months; mOS: 25.5-26.3 months; ORR: not reported) [40,53] and patients from often under-represented ethnic groups (e.g., Asian populations: mPFS: 7.4-14.1 months; mOS: 16.5-33.2 months; ORR: 21-59\%) [44,45,49,51,55,56,58,59] were included in the analysis. Overall, efficacy outcomes in these populations appeared to be consistent with the overall population. 


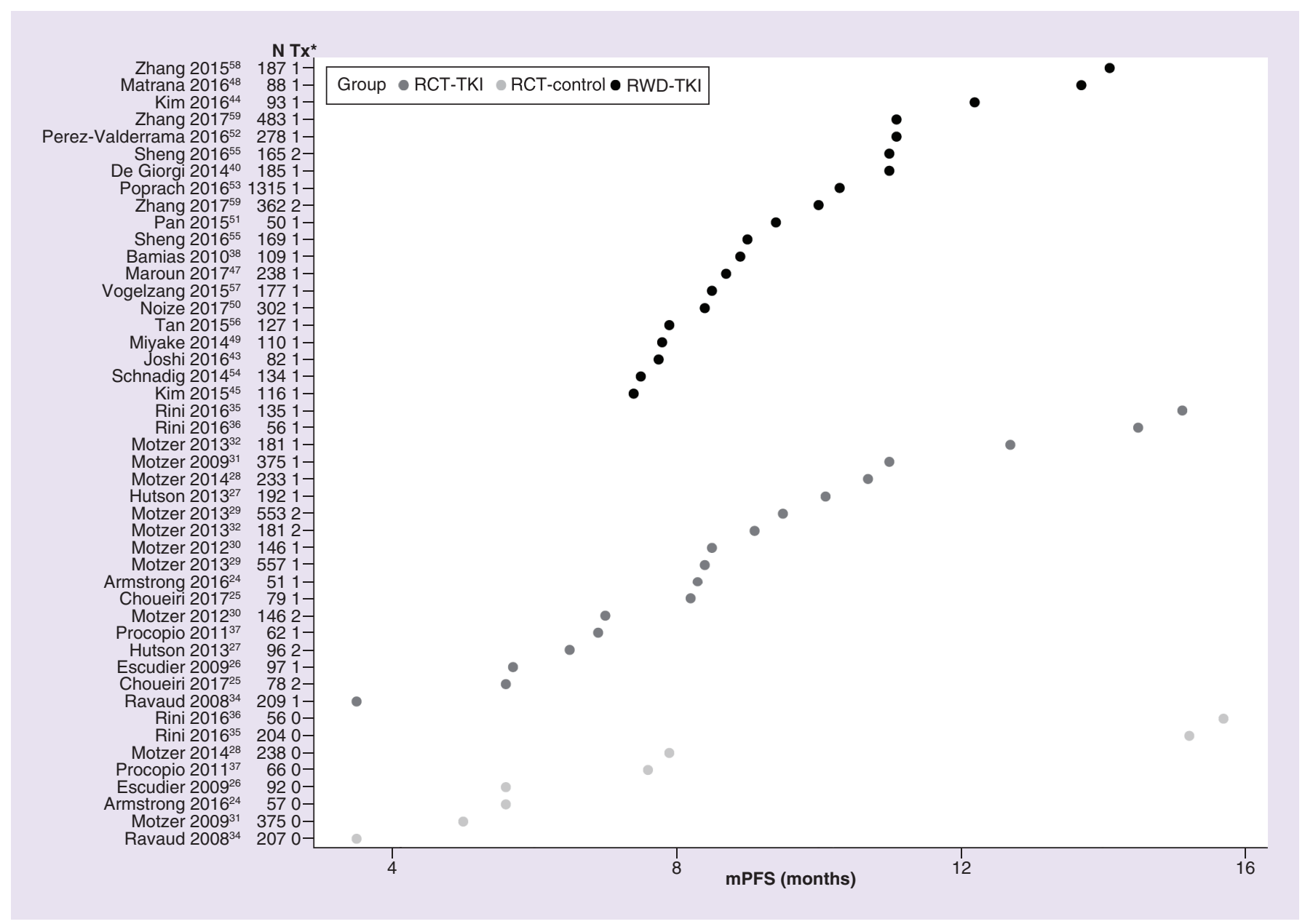

Figure 2. Observed median progression-free survival data.

* 1 or 2 denotes TKI treatment group, 0 denotes non-TKI treatment group.

mPFS: Median progression-free survival; RCT-control: Matching control, non-TKI treatments in randomized controlled trial; RCT-TKI: TKI as a single agent in the first-line setting in randomized controlled trials; RWD-TKI: TKI as a single agent in the first-line setting in real-world data study; TKI: Tyrosine kinase inhibitor; Tx: Treatment group.

Study heterogeneity was assessed as high and was accounted for in the modeling. RCT data tended to show a greater degree of between-study heterogeneity than RWD for each efficacy end point. The impact of patient age and duration of therapy on efficacy end points is shown in Supplementary Figures 5, 6 and 7. Patient age had no effect on mPFS or mOS (Supplementary Figures 5A \& 6A); however, there was a small increase in ORR as patient age increased (Supplementary Figure 7A). As the duration of therapy increased so did mPFS, mOS and ORR (Supplementary Figures 5B, 6B \& S7B).

\section{Discussion}

The stringent eligibility criteria of RCTs are an essential feature that ensures high internal validity; however, this may limit external validity, as RCT populations may not reflect the diverse population seen by clinicians in the real-world setting. This raises the question of whether RCT results are generalizable in different clinical settings $[1,60]$. This large systematic review and meta-analysis aimed to assess how efficacy outcomes from RWD compare with those from RCTs in patients with $\mathrm{mRCC}$ receiving first-line TKI therapy.

Overall, mPFS, mOS and ORR were similar between RCTs and RWD. Our results were largely consistent with previously reported analyses [8,61-64]. A systematic review of sunitinib RWD in the UK, which compared RWD with RCTs within groups of patients with similar characteristics, found that mPFS (6.7-11.1 months) was comparable with data from RCTs [8]. Our analysis found that the overall estimate of mPFS did not differ significantly between 


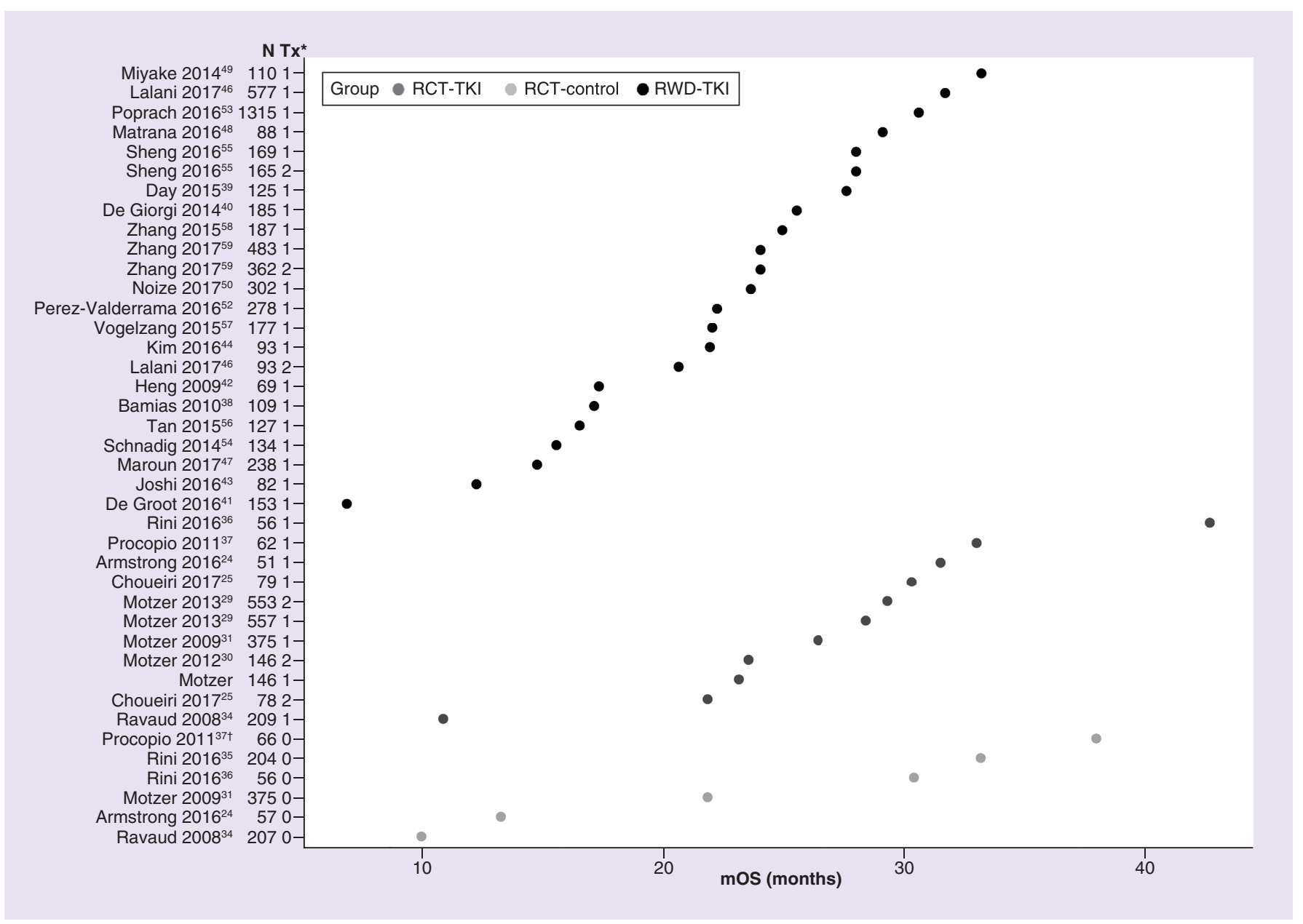

Figure 3. Observed median overall survival data.

* 1 or 2 denotes TKI treatment group, 0 denotes non-TKI treatment group.

**mOS data were not mature at the time of publication and were extracted from a later analysis [33].

mOS: Median overall survival; RCT-control: Matching control, non-TKI treatments in randomized controlled trial; RCT-TKI: TKI agent in randomized controlled trial; RWD-TKI: TKI as a single agent in the first-line setting in real-world data study; TKI: Tyrosine kinase inhibitor; Tx: Treatment group.

RCTs and RWD; however, RCTs reporting mPFS had a wider range of values than RWD data. This may have been partly attributable to a potential time bias and inconsistent use of Response Evaluation Criteria in Solid Tumors (RECIST) among studies. The method of assessment for mPFS and ORR was not consistently reported across RWD and RCTs. The method of assessment is likely to differ between RWD and RCTs by design. For example, the timing of scans is less proscriptive and likely to be less frequent in RWD studies and this may particularly affect the reported PFS. Dependent on the source of RWD, it is likely that mPFS and ORR were assessed unblinded by the clinician, whereas RCTs may employ blinded independent review to assess disease-progression events.

An analysis of RWD from the Renal Cell Carcinoma Outcomes Research Dataset (RECCORD) registry reported that in patients receiving sunitinib, pazopanib or sorafenib as first-line treatment, mOS was 23.9 months and consistent with data reported in clinical trials [61]. In our analysis, mOS was approximately 6 months longer in RCTs compared with RWD. Although perhaps clinically meaningful, this difference did not reach statistical significance. Other studies have reported substantial variability in RWD for OS, which may be due to a wider range of patient age, comorbidities, performance status and disease risk found in RWD compared with RCTs [8,62]. A US-based cohort study found patients with mRCC seen in routine clinical practice were more likely to have poor-risk disease by Memorial Sloan Kettering Cancer Center criteria and impaired performance status (Eastern Cooperative Oncology Group) compared with patients enrolled in Phase III TKI trials [62]. An estimated 35-57\% 


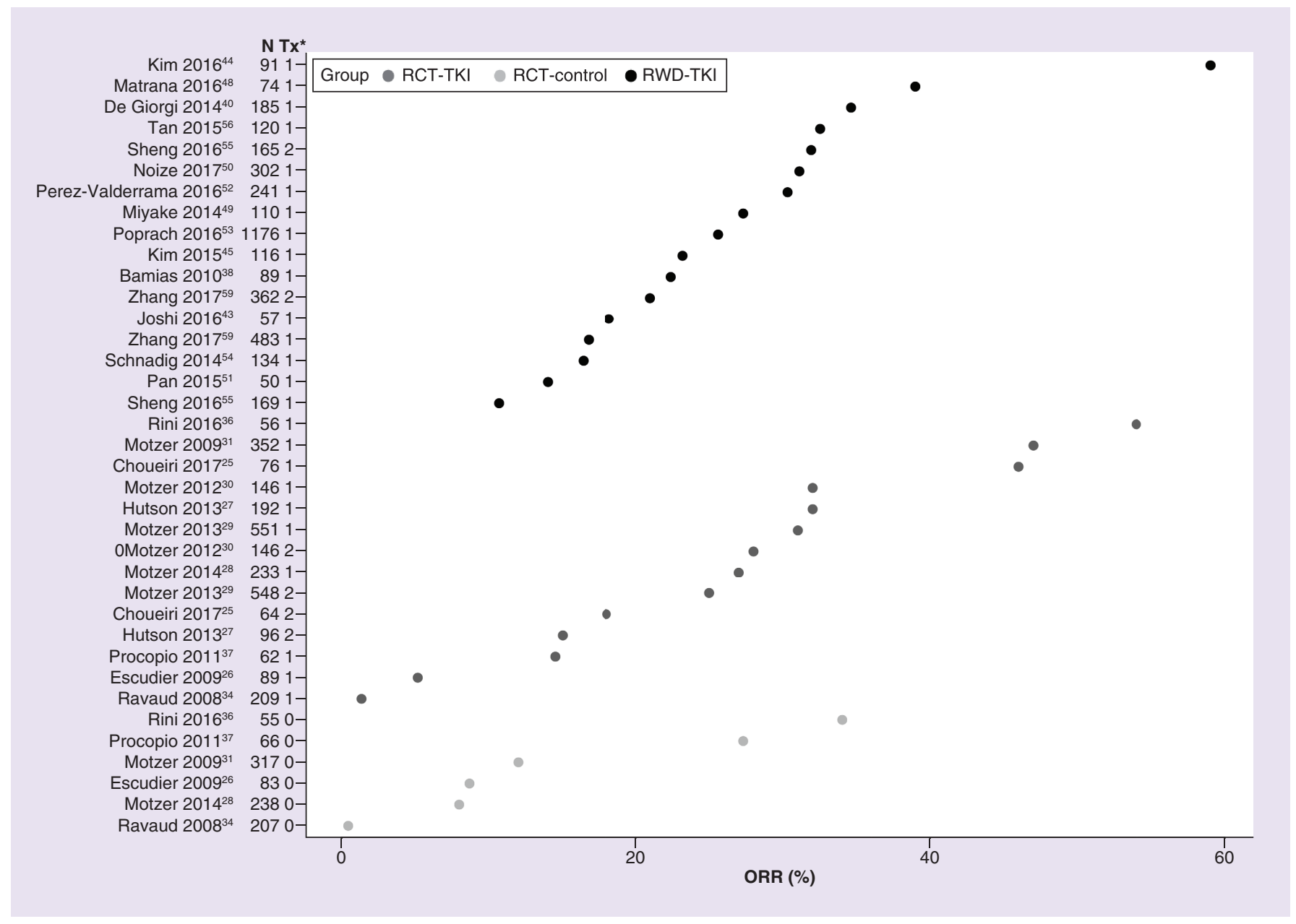

Figure 4. Observed objective response rate data.

*1 or 2 denotes TKI treatment group, 0 denotes non-TKI treatment group.

ORR: Objective response rate; RCT-control: Matching control, non-TKI treatments in randomized controlled trial; RCT-TKI: TKI agent in randomized controlled trial; RWD-TKI: TKI as a single agent in the first-line setting in real-world data study; TKI: Tyrosine kinase inhibitor; Tx: Treatment group.

of patients with $\mathrm{mRCC}$ seen in routine clinical practice are ineligible for clinical trials [62,65-67]. Patients who do not meet eligibility criteria for clinical trials may have inferior survival outcomes than those reported in RCTs [65]. Poor performance status and presence of brain metastases are the most common reasons these patients failed to meet eligibility criteria [62,65-67]. Notably, the American Society of Clinical Oncology and Friends of Cancer Research have recently submitted draft guidance to the FDA to broaden cancer clinical trial eligibility criteria, including patients with brain metastases and prior/concurrent malignancies [13]. A sunitinib global-expanded access trial, which included patients with mRCC ineligible for RCTs, found mPFS was shorter in patients with poor performance status (3.5 months) or brain metastases (5.3 months) compared with the overall population (9.4 months) [68]. However, these outcomes still represented a clinically meaningful benefit and these groups of patients had a safety profile similar to the overall population [68].

It is well known that demographic factors can influence treatment outcomes. An analysis of submissions to the FDA from 2008 to 2013 for new cancer-specific therapeutic products revealed many demographic subgroups are under-represented in RCTs, including ethnic groups and elderly patients [69]. Ethnicity has been reported to affect both the incidence and survival outcomes of patients with RCC [70] and increasing age is considered to be an adverse prognostic factor associated with higher tumor stage [71]. Under-representation of these patient groups makes it difficult for clinicians to extrapolate data from RCTs in order to make clinical decisions. Our analysis included RWD studies of both elderly patients $[40,53]$ and patients from under-represented ethnic groups (e.g., Asian 
(A)

mPFS (95\% Cl), months

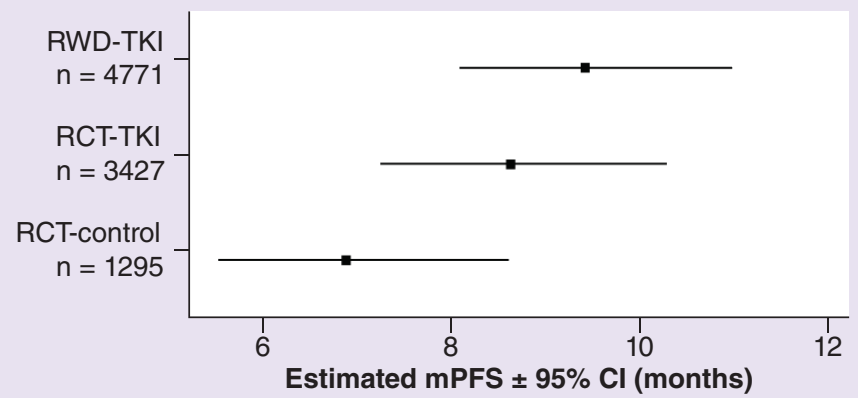

$9.4(8.1-11.0)$

$8.6(7.3-10.3)$

$6.9(5.5-8.6)$

(B)

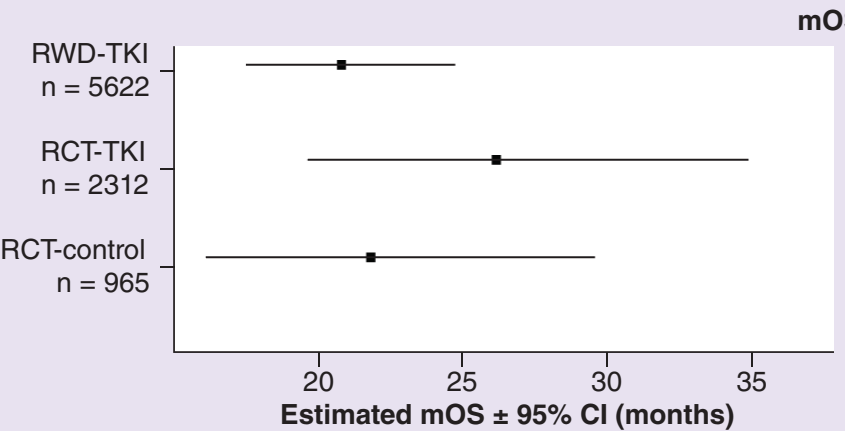

mOS $(95 \% \mathrm{Cl})$, months

$20.8(17.5-24.8)$

$26.2(19.6-35.0)$

$21.8(16.1-29.7)$

(C)

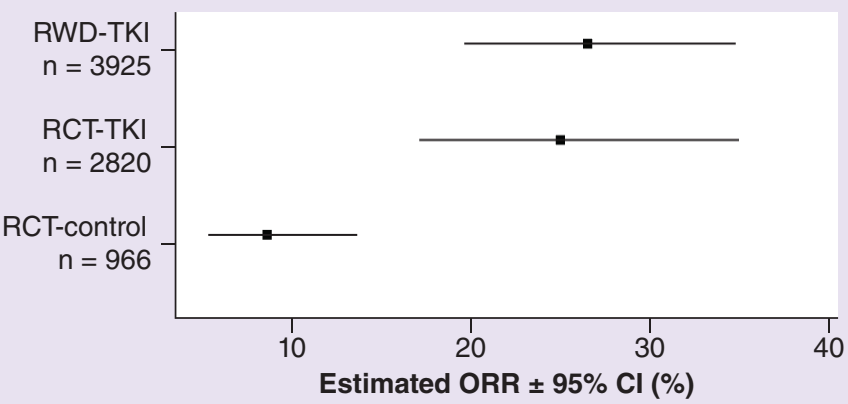

ORR (95\% Cl), \%

26.5 (19.6-34.8)

$25.0(17.1-35.0)$

$8.5(5.2-13.6)$

Figure 5. Meta-analysis efficacy estimates. Forest plot of (A) mPFS, (B) mOS and (C) ORR for patients with mRCC treated with first-line TKIs.

mOS: Median overall survival; mPFS: Median progression-free survival; mRCC: Metastatic renal cell carcinoma; ORR: Objective response rate; RCT: Randomized controlled trial; RCT-control: Matching control, non-TKI in randomized controlled trial; RCT-TKI: TKI agent in randomized controlled trial; RWD: Real-world data; RWD-TKI: TKI agent in RWD study; TKI: Tyrosine kinase inhibitor.

populations) [44,45,49,51,55,56,58,59]. Although no subgroup analyses were performed for these patient groups, efficacy outcomes appeared to be generally comparable with the overall estimates of the younger, predominantly white populations found in RCTs.

Since 2005, the dramatic increase in targeted treatment options for mRCC has resulted in substantial improvements in health and survival [72,73]. The recent development of immune checkpoint inhibitors and their use in combination with TKIs, such as avelumab or pembrolizumab plus axitinib, look set to provide another leap forward in the treatment of mRCC [74,75]. As more treatment options become available, it is increasingly complex for clinicians to select the optimal course of treatment for patients; efficacy, treatment sequence, treatment combination and toxicity profiles must all be considered and weighed against the unique characteristics of each patient. Of the patients who receive first-line treatment, $47 \%$ receive second-line treatment and $17 \%$ go on to receive third- or 
even subsequent-line treatment [76]. The optimal sequence of mRCC treatment is an ongoing debate [77]. The time and resources required to conduct RCTs make it impossible to complete comparative trials for all relevant medical interventions in the appropriate populations. Although published treatment algorithms can help refine options, RWD have the potential to further clarify the most appropriate course of treatment [78].

The influence of RWD on clinical practice appears to be increasing [79]. Evidence from RWD can help inform cost-analysis, dosing, effectiveness, and safety outcomes and label expansion [2]. Furthermore, there is growing interest in utilizing RWD to aid the approval of new treatments [80]. Importantly, the increased use of electronic health records, among other technologies, means that RWD are readily available. Compared with RCTs, knowledge gained from RWD can be acquired faster and with less expense [2]. However, RWD must be interpreted carefully, as it may differ from RCT data under alternative disease and treatment conditions. Diligent planning and study design are essential to ensure the validity of RWD in that variations in data quality, consistency and confounding remain major issues in the widespread use of RWD [5]. Guidelines have been published to improve the standard of reported RWD, including the REporting of studies Conducted using Observational Routinely-collected Data (RECORD) and STrengthening the Reporting of OBservational studies in Epidemiology (STROBE) [81,82]. However, variation in the quality of RWD reporting remains a concern [83]. Improvements are needed in both the design and data-quality assurance of RWD to provide valid, reliable studies [5].

Limitations to our study include a potential time bias across efficacy end points for RCTs, high between-study heterogeneity and a larger variety of TKIs investigated in RCTs compared with RWD studies. Not all TKIs included in this analysis subsequently gained regulatory approval or recommendation as first-line treatment for mRCC. Of the investigated TKIs, sunitinib, pazopanib, tivozanib and cabozantinib are the only TKIs currently recommended as first-line treatment for favorable-risk mRCC $[15,17,18]$. One TKI study included in the analysis was a Phase III study of lapatinib [34]. Lapatinib, an inhibitor of EGFR2, is no longer used in the treatment of mRCC. Of the reported RCT efficacy measures, the lowest mPFS and mOS were from the lapatinib study, with values of 3.5 and 10.8 months, respectively. Overall estimates of RCT efficacy end points may therefore be slightly underestimated. Other classes of first-line treatment are now available for mRCC, but did not meet inclusion criteria for this analysis. Moreover, our analysis only included TKIs as single agents in first-line mRCC treatment and not their use in combination with other agents. The recent approvals of the immune checkpoint inhibitor combination nivolumab plus ipilimumab and the TKI plus immune checkpoint inhibitor combinations (avelumab plus axitinib and pembrolizumab plus axitinib) may limit the long-term applicability of this analysis, as these treatments transition to be the standard of care. Results for mPFS and ORR should be interpreted conservatively due to between-study differences in assessment time points and inconsistent use, or reporting of, RECIST-based evaluation. Our study was not designed to examine specific patient subgroups of interest; however, further analyses of patient subgroups and comparative analyses of first-line mRCC treatment effectiveness and safety would be of clinical value. Future RWD studies should be specifically designed to answer clinical questions not supported by RCTs.

\section{Conclusion}

These analyses demonstrated that RWD studies validate efficacy-based outcomes from RCTs. RWD studies can provide supplementary information from groups of patients who may not be eligible for RCTs, such as patients with brain metastases or ECOG $>1$, and will therefore aid in clinical decisions. The data presented support an increased role for the use of RWD outputs in understanding the treatment of patients with mRCC by complementing the evidence produced from RCTs.

\section{Future perspective}

The FDA has recently released draft guidance documents that aim to broaden the eligibility criteria of RCTs for new cancer treatments [84-87]. In the next 5-10 years, we will likely see less restrictive eligibility criteria for patients with brain metastases, pediatric patients, patients with organ dysfunction or pervious cancers, and patients with HIV, or hepatitis B or C. This will improve the generalizability of RCTs and may also lead to an increase in the proportion of patients enrolled in cancer RCTs. In parallel, we will likely see an increase in the number and quality of RWD studies. The recent approval of nivolumab plus ipilimumab, avelumab plus axitinib, and pembrolizumab plus axitinib is set to transform the treatment of mRCC; however, it would not be possible to conduct a sufficient number of RCTs to determine the best combination for first-line treatment, or the optimal sequence of treatments 
if patients progress to subsequent-line treatment. RWD may provide complementary evidence to aid clinicians in choosing the most appropriate combination and sequence of mRCC treatments.

As the quality of RWD studies improves, the influence of RWD on healthcare policy decisions and drug development is also likely to increase. Despite an increasing number of studies showing consistency with results from RCTs and RWD sources, even robust RWD studies are unlikely to replace the RCT. Instead, RWD will continue to be seen as complementary to RCTs. Nevertheless, both the FDA and the EMA are preparing to publish guidance on the use of RWD in supporting therapeutic indications. The effective use of RWD is likely to expedite the approval of new therapeutic indications.

\section{Summary points}

\section{Background}

- Randomized controlled trials (RCTs) are the gold standard for gaining regulatory approval of new medical interventions. However, RCT populations may not fully represent those of everyday clinical practice.

- Real-world data (RWD) could provide evidence for patients not eligible for RCTs, such as patients with brain metastases.

- The introduction of targeted therapy, including tyrosine kinase inhibitors (TKIs), revolutionized the treatment of metastatic renal cell carcinoma.

- This systematic review and meta-analysis addressed how efficacy outcomes from RWD can support those from RCTs, in the context of TKIs as first-line treatment of metastatic renal cell carcinoma.

Search results

- A total of 35 studies (13 RCTs and 22 RWD) met eligibility criteria, and 31, 28 and 25 studies, respectively, reported summary median progression-free survival, median overall survival and objective response rate results based on 8493,8899 and 7711 patients.

Efficacy of meta-analysis

- Overall, median progression-free survival was numerically longer in RWD versus RCT (9.4 [95\% Cl: 8.1-11.0] vs 8.6 [7.3-10.3] months; $p=0.4505)$; median overall survival was numerically shorter in RWD versus RCTs (20.8 [17.5-24.8] months vs 26.2 [19.6-35.0]; $\mathrm{p}=0.1643$ ); and objective response rate was similar in RWD versus RCTs (26.5\% [19.6-34.8] vs $25.0 \%$ [17.1-35.0]; $p=0.7990)$; however, these differences did not reach statistical significance.

- There was evidence of a potential time bias across all outcome measures, but no evidence of publication bias.

Conclusion

- This study supports the use of RWD as a supportive source of evidence that can be used to inform clinical decisions.

Financial \& competing interests disclosure

M Moran, D Nickens and M Bennetts are employees of Pfizer, Inc. K Adcock was an employee of Pfizer between September 2017 and 2018 (undergraduate program). N Charnley has received honoraria for lecture fees and advisory boards from Pfizer, Ipsen and Janssen. K Fife has received honoraria for advice or lecture fees from BMS, Pfizer, Esai, Novartis and Roche. The authors have no other relevant affiliations or financial involvement with any organization or entity with a financial interest in or financial conflict with the subject matter or materials discussed in the manuscript apart from those disclosed.

This study was sponsored by Pfizer, Inc. Medical writing support was provided by L Adams, of Engage Scientific Solutions, and was funded by Pfizer.

\section{Data sharing statement}

Upon request, and subject to certain criteria, conditions and exceptions (https://www.pfizer.com/science/clinical-trials/trial-data-an d-results for more information), Pfizer will provide access to individual de-identified participant data from Pfizer-sponsored global interventional clinical studies conducted for medicines, vaccines and medical devices for indications that have been approved in the USA and/or the EU or; in programs that have been terminated (i.e., development for all indications has been discontinued). Pfizer will also consider requests for the protocol, data dictionary and statistical analysis plan. Data may be requested from Pfizer trials 24 months after study completion. The de-identified participant data will be made available to researchers whose proposals meet the research criteria and other conditions, and for which an exception does not apply, via a secure portal. To gain access, data requesters must enter into a data access agreement with Pfizer. 
Open access

This work is licensed under the Attribution-NonCommercial-NoDerivatives 4.0 Unported License. To view a copy of this license, visit http://creativecommons.org/licenses/by-nc-nd/4.0/

\section{References}

Papers of special note have been highlighted as: $\bullet$ of interest

1. Rothwell PM. Commentary: external validity of results of randomized trials: disentangling a complex concept. Int. J. Epidemiol. 39(1), 94-96 (2010).

2. Sherman RE, Anderson SA, Dal Pan GJ et al. Real-world evidence - what is it and what can it tell us? N. Engl. J. Med. 375(23), 2293-2297 (2016).

3. Rothwell PM. External validity of randomised controlled trials: 'to whom do the results of this trial apply?'. Lancet 365(9453), 82-93 (2005).

- A review of the key factors underlying the external validity of randomized controlled trials.

4. Van Spall HG, Toren A, Kiss A, Fowler RA. Eligibility criteria of randomized controlled trials published in high-impact general medical journals: a systematic sampling review. JAMA 297(11), 1233-1240 (2007).

5. Khozin S, Blumenthal GM, Pazdur R. Real-world data for clinical evidence generation in oncology. J. Natl Cancer Inst. 109(11), (2017). doi:10.1093/jnci/djx187

6. Association of the British Pharmaceutical Industry. The vision for real world data - harnessing the opportunities in the UK (2011). http://www.abpi.org.uk/publications/vision-for-real-world-data

7. McDonald L, Lambrelli D, Wasiak R, Ramagopalan SV. Real-world data in the United Kingdom: opportunities and challenges. BMC Med. 14(1), 97 (2016).

8. Argyropulo-Palmer M, Jenkins A, Theti DS, Larkin J, Montgomery D. Sunitinib in metastatic renal cell carcinoma: a systematic review of UK real world data. Front. Oncol. 5, 195 (2015).

- Previous systematic review showing that median progression-free survival and reported adverse events were similar in real-world studies and randomized controlled trials.

9. Katkade VB, Sanders KN, Zou KH. Real world data: an opportunity to supplement existing evidence for the use of long-established medicines in health care decision making. J. Multidiscip. Healthc. 11, 295-304 (2018).

10. Murthy VH, Krumholz HM, Gross CP. Participation in cancer clinical trials: race-, sex-, and age-based disparities. JAMA 291(22), 2720-2726 (2004).

11. Dias AL, Chao JH, Lee D, Wu Y, Kloecker GH. Patient perceptions concerning clinical trials in oncology patients. Contemp. Clin. Trials Commun. 4, 179-185 (2016).

12. Schroen AT, Petroni GR, Wang $\mathrm{H}$ et al. Preliminary evaluation of factors associated with premature trial closure and feasibility of accrual benchmarks in Phase III oncology trials. Clin. Trials 7(4), 312-321 (2010).

13. American Society of Clinical Oncology (ASCO) and Friends of Cancer Research. Cancer clinical trial eligibility criteria (2018). https: //www.focr.org/sites/default/files/pdf/ASCO-Friends\%20Cancer\%20Clinical\%20Trials\%20Eligibility\%20Criteria_Aug_8_2018_0.pdf

14. Bray F, Ferlay J, Soerjomataram I, Siegel RL, Torre LA, Jemal A. Global cancer statistics 2018: GLOBOCAN estimates of incidence and mortality worldwide for 36 cancers in 185 countries. CA Cancer J. Clin. 68(6), 394-424 (2018).

15. National Comprehensive Cancer Network. NCCN clinical practice guidelines in oncology: kidney cancer. Version 3 (2019). https://www.nccn.org/professionals/physician_gls/def ault.aspx\#site

16. Dabestani S, Thorstenson A, Lindblad P, Harmenberg U, Ljungberg B, Lundstam S. Renal cell carcinoma recurrences and metastases in primary non-metastatic patients: a population-based study. World J. Urol. 34(8), 1081-1086 (2016).

17. Escudier B, Porta C, Schmidinger M et al. Renal cell carcinoma: ESMO Clinical Practice Guidelines for diagnosis, treatment and follow-up. Ann. Oncol. 30(5), 706-720 (2019).

18. European Association of Urology. Renal cell carcinoma guidelines (2019). https://uroweb.org/guideline/renal-cell-carcinoma/

19. The American Cancer Society. Survival rates for kidney cancer by stage (31 January 2019). www.cancer.org/cancer/kidney-cancer/detection-diagnosis-staging/survival-rates.html

20. Zarrabi K, Fang C, Wu S. New treatment options for metastatic renal cell carcinoma with prior anti-angiogenesis therapy. J. Hematol. Oncol. 10(1), 38 (2017).

21. Liberati A, Altman DG, Tetzlaff J et al. The PRISMA statement for reporting systematic reviews and meta-analyses of studies that evaluate health care interventions: explanation and elaboration. J. Clin. Epidemiol. 62(10), e1-34 (2009).

22. Morrison A, Polisena J, Husereau D et al. The effect of English-language restriction on systematic review-based meta-analyses: a systematic review of empirical studies. Int. J. Technol. Assess. Health Care 28(2), 138-144 (2012).

23. Moran M, Nickens D, Adcock K et al. Sunitinib for metastatic renal cell carcinoma: a systematic review and meta-analysis of real-world and clinical trials data. Target Oncol. 14(4), 405-416 (2019). 
24. Armstrong AJ, Halabi S, Eisen T et al. Everolimus versus sunitinib for patients with metastatic non-clear cell renal cell carcinoma (ASPEN): a multicentre, open-label, randomised Phase II trial. Lancet Oncol. 17(3), 378-388 (2016).

25. Choueiri TK, Halabi S, Sanford BL et al. Cabozantinib versus sunitinib as initial targeted therapy for patients with metastatic renal cell carcinoma of poor or intermediate risk: The Alliance A031203 CABOSUN Trial. J. Clin. Oncol. 35(6), 591-597 (2017).

26. Escudier B, Szczylik C, Hutson TE et al. Randomized Phase II trial of first-line treatment with sorafenib versus interferon $\alpha$-2a in patients with metastatic renal cell carcinoma. J. Clin. Oncol. 27(8), 1280-1289 (2009).

27. Hutson TE, Lesovoy V, Al-Shukri S et al. Axitinib versus sorafenib as first-line therapy in patients with metastatic renal-cell carcinoma: a randomised open-label Phase III trial. Lancet Oncol. 14(13), 1287-1294 (2013).

28. Motzer RJ, Barrios CH, Kim TM et al. Phase II randomized trial comparing sequential first-line everolimus and second-line sunitinib versus first-line sunitinib and second-line everolimus in patients with metastatic renal cell carcinoma. J. Clin. Oncol. 32(25), 2765-2772 (2014).

29. Motzer RJ, Hutson TE, Cella D et al. Pazopanib versus sunitinib in metastatic renal-cell carcinoma. N. Engl. J. Med. 369(8), $722-731$ (2013).

30. Motzer RJ, Hutson TE, Olsen MR et al. Randomized Phase II trial of sunitinib on an intermittent versus continuous dosing schedule as first-line therapy for advanced renal cell carcinoma. J. Clin. Oncol. 30(12), 1371-1377 (2012).

31. Motzer RJ, Hutson TE, Tomczak $\mathrm{P}$ et al. Overall survival and updated results for sunitinib compared with interferon alfa in patients with metastatic renal cell carcinoma. J. Clin. Oncol. 27(22), 3584-3590 (2009).

32. Motzer RJ, Nosov D, Eisen T et al. Tivozanib versus sorafenib as initial targeted therapy for patients with metastatic renal cell carcinoma: results from a Phase III trial. J. Clin. Oncol. 31(30), 3791-3799 (2013).

33. Procopio G, Verzoni E, Bracarda S et al. Overall survival for sorafenib plus interleukin-2 compared with sorafenib alone in metastatic renal cell carcinoma (mRCC): final results of the ROSORC trial. Ann. Oncol. 24(12), 2967-2971 (2013).

34. Ravaud A, Hawkins R, Gardner JP et al. Lapatinib versus hormone therapy in patients with advanced renal cell carcinoma: a randomized Phase III clinical trial. J. Clin. Oncol. 26(14), 2285-2291 (2008).

35. Rini BI, Stenzl A, Zdrojowy R et al. IMA901, a multipeptide cancer vaccine, plus sunitinib versus sunitinib alone, as first-line therapy for advanced or metastatic renal cell carcinoma (IMPRINT): a multicentre, open-label, randomised, controlled, Phase III trial. Lancet Oncol. 17(11), 1599-1611 (2016).

36. Rini BI, Tomita Y, Melichar B et al. Overall survival analysis from a randomized Phase II study of axitinib with or without dose titration in first-line metastatic renal cell carcinoma. Clin. Genitourin. Cancer 14(6), 499-503 (2016).

37. Procopio G, Verzoni E, Bracarda $S$ et al. Sorafenib with interleukin-2 vs sorafenib alone in metastatic renal cell carcinoma: the ROSORC trial. Br. J. Cancer 104(8), 1256-1261 (2011).

38. Bamias A, Karadimou A, Lampaki S et al. Prognostic stratification of patients with advanced renal cell carcinoma treated with sunitinib: comparison with the Memorial Sloan-Kettering prognostic factors model. BMC Cancer 10, 45 (2010).

39. Day D, Kanjanapan Y, Kwan E et al. Patterns of care for metastatic renal cell carcinoma in Australia. BJU Int. 116(Suppl. 3), 36-41 (2015).

40. De Giorgi U, Scarpi E, Sacco C et al. Standard vs adapted sunitinib regimen in elderly patients with metastatic renal cell cancer: results from a large retrospective analysis. Clin. Genitourin. Cancer 12(3), 182-189 (2014).

41. De Groot S, Sleijfer S, Redekop WK et al. Variation in use of targeted therapies for metastatic renal cell carcinoma: results from a Dutch population-based registry. BMC Cancer 16, 364 (2016).

42. Heng DY, Chi KN, Murray N et al. A population-based study evaluating the impact of sunitinib on overall survival in the treatment of patients with metastatic renal cell cancer. Cancer 115(4), 776-783 (2009).

43. Joshi A, Ramaswamy $\mathrm{A}$, Noronha $\mathrm{V}$ et al. Efficacy and safety of sorafenib in advanced renal cell cancer and validation of Heng criteria. Indian J. Cancer 53(3), 423-428 (2016).

44. Kim MJ, Park SH, Lee JL, Lee SH, Lee SJ, Lim HY. A Korean multi-center, real-world, retrospective study of first-line pazopanib in unselected patients with metastatic renal clear-cell carcinoma. BMC Urol. 16(1), 46 (2016).

45. Kim SH, Kim S, Nam BH et al. Efficacy and safety of sorafenib therapy on metastatic renal cell carcinoma in Korean patients: results from a retrospective multicenter study. PLoS ONE 10(8), e0135165 (2015).

46. Lalani AA, Li H, Heng DYC et al. First-line sunitinib or pazopanib in metastatic renal cell carcinoma: the Canadian experience. Can. Urol. Assoc. J. 11(3-4), 112-117 (2017).

47. Maroun R, Fleury L, Nachbaur G, Maunoury F, Vanhille JL, Durand-Zaleski I. Real-world costs and outcomes in metastatic renal cell carcinoma patients treated with targeted therapies: a cohort study from the French health insurance database. Curr. Med. Res. Opin. 33(10), 1755-1762 (2017).

48. Matrana MR, Bathala T, Campbell MT et al. Outcomes of unselected patients with metastatic clear-cell renal cell carcinoma treated with first-line pazopanib therapy followed by vascular endothelial growth factor receptor tyrosine kinase inhibitors or mammalian target of rapamycin inhibitors: a single institution experience. BJU Int. 118(2), 264-271 (2016). 
49. Miyake H, Miyazaki A, Harada K, Fujisawa M. Assessment of efficacy, safety and quality of life of 110 patients treated with sunitinib as first-line therapy for metastatic renal cell carcinoma: experience in real-world clinical practice in Japan. Med. Oncol. 31(6), 978 (2014).

50. Noize P, Grelaud A, Bay JO et al. Real-life patterns of use, safety and effectiveness of sunitinib in first-line therapy of metastatic renal cell carcinoma: the SANTORIN cohort study. Pharmacoepidemiol. Drug Saf. 26(12), 1561-1569 (2017).

51. Pan X, Huang H, Huang Y et al. Sunitinib dosing schedule $2 / 1$ improves tolerability, efficacy, and health-related quality of life in Chinese patients with metastatic renal cell carcinoma. Urol. Oncol. 33(6), 268 (2015).e269-215

52. Perez-Valderrama B, Arranz Arija JA, Rodriguez Sanchez A et al. Validation of the International Metastatic Renal-Cell Carcinoma Database Consortium (IMDC) prognostic model for first-line pazopanib in metastatic renal carcinoma: the Spanish Oncologic Genitourinary Group (SOGUG) SPAZO study. Ann. Oncol. 27(4), 706-711 (2016).

53. Poprach A, Lakomy R, Bortlicek $\mathrm{Z}$ et al. Efficacy of sunitinib in elderly patients with metastatic renal cell carcinoma: data from real-world clinical practice. Drugs Aging 33(9), 655-663 (2016).

54. Schnadig ID, Hutson TE, Chung $\mathrm{H}$ et al. Dosing patterns, toxicity, and outcomes in patients treated with first-line sunitinib for advanced renal cell carcinoma in community-based practices. Clin. Genitourin. Cancer 12(6), 413-421 (2014).

55. Sheng X, Chi Z, Cui C et al. Efficacy and safety of sorafenib versus sunitinib as first-line treatment in patients with metastatic renal cell carcinoma: largest single-center retrospective analysis. Oncotarget 7(19), 27044-27054 (2016).

56. Tan HS, Li H, Hong YW et al. Efficacy and safety of an attenuated-dose sunitinib regimen in metastatic renal cell carcinoma: results from a prospective registry in Singapore. Clin. Genitourin. Cancer 13(4), e285-e295 (2015).

57. Vogelzang NJ, Hackshaw MD, Hutson TE et al. First-line and sequential use of pazopanib followed by mammalian target of rapamycin inhibitor therapy among patients with advanced renal cell carcinoma in a US community oncology setting. Clin. Genitourin. Cancer 13(3), 210-217 (2015).

58. Zhang HL, Qin XJ, Wang HK et al. Clinicopathological and prognostic factors for long-term survival in Chinese patients with metastatic renal cell carcinoma treated with sorafenib: a single-center retrospective study. Oncotarget 6(34), 36870-36883 (2015).

59. Zhang HL, Sheng XN, Li XS et al. Sorafenib versus sunitinib as first-line treatment agents in Chinese patients with metastatic renal cell carcinoma: the largest multicenter retrospective analysis of survival and prognostic factors. BMC Cancer 17(1), 16 (2017).

60. Dekkers OM, von Elm E, Algra A, Romijn JA, Vandenbroucke JP. How to assess the external validity of therapeutic trials: a conceptual approach. Int. J. Epidemiol. 39(1), 89-94 (2010).

61. Wagstaff J, Jones R, Hawkins R et al. Treatment patterns and clinical outcomes in patients with renal cell carcinoma in the UK: insights from the RECCORD registry. Ann. Oncol. 27(1), 159-165 (2016).

62. Mitchell AP, Harrison MR, Walker MS, George DJ, Abernethy AP, Hirsch BR. Clinical trial participants with metastatic renal cell carcinoma differ from patients treated in real-world practice. J. Oncol. Pract. 11(6), 491-497 (2015).

- A retrospective analysis shows how patients with metastatic renal cell carcinoma treated in everyday clinical practice are sicker than those enrolled in clinical trials.

63. Harrison MR, Hirsch BR, George DJ et al. Real-world outcomes in metastatic renal cell carcinoma: insights from a Joint Community-Academic Registry. J. Oncol. Pract. 10(2), e63-72 (2014).

64. Ruiz-Morales JM, Swierkowski M, Wells JC et al. First-line sunitinib versus pazopanib in metastatic renal cell carcinoma: results from the International Metastatic Renal Cell Carcinoma Database Consortium. Eur. J. Cancer 65, 102-108 (2016).

65. Heng DY, Choueiri TK, Rini BI et al. Outcomes of patients with metastatic renal cell carcinoma that do not meet eligibility criteria for clinical trials. Ann. Oncol. 25(1), 149-154 (2014).

- The research highlights how patients with metastatic renal cell carcinoma are ineligible for clinical trials have inferior survival outcomes compared with patients enrolled in clinical trials.

66. Nieder C, Syed MA, Dalhaug A, Pawinski A, Norum J. Eligibility for Phase III clinical trials of systemic therapy in real-world patients with metastatic renal cell cancer managed in a rural region. Med. Oncol. 34(9), 149 (2017).

67. Marschner N, Staehler M, Muller L et al. Survival of patients with advanced or metastatic renal cell carcinoma in routine practice differs from that in clinical trials-analyses from the German Clinical RCC Registry. Clin. Genitourin. Cancer 15(2), e209-e215 (2017).

68. Gore ME, Szczylik C, Porta C et al. Final results from the large sunitinib global expanded-access trial in metastatic renal cell carcinoma. Br. J. Cancer 113(1), 12-19 (2015).

69. Ramamoorthy A, Knepper TC, Merenda C et al. Demographic composition of select oncologic new molecular entities approved by the FDA between 2008 and 2017. Clin. Pharmacol. Ther. 104(5), 940-948 (2018).

- The study describes the demographic profiles of patients enrolled in randomized controlled trials for new therapeutic products and concludes that many patient sub-groups are under-represented.

70. Stafford HS, Saltzstein SL, Shimasaki S, Sanders C, Downs TM, Sadler GR. Racial/ethnic and gender disparities in renal cell carcinoma incidence and survival. J. Urol. 179(5), 1704-1708 (2008).

71. Jung EJ, Lee HJ, Kwak C, Ku JH, Moon KC. Young age is independent prognostic factor for cancer-specific survival of low-stage clear cell renal cell carcinoma. Urology 73(1), 137-141 (2009). 
72. Li P, Wong YN, Armstrong $\mathrm{K}$ et al. Survival among patients with advanced renal cell carcinoma in the pretargeted versus targeted therapy eras. Cancer Med. 5(2), 169-181 (2016).

73. De Groot S, Blommestein HM, Redekop WK et al. Potential health gains for patients with metastatic renal cell carcinoma in daily clinical practice: a real-world cost-effectiveness analysis of sequential first- and second-line treatments. PLoS ONE 12(5), e0177364 (2017).

74. Motzer RJ, Penkov K, Haanen J et al. Avelumab plus axitinib versus sunitinib for advanced renal-cell carcinoma. N. Engl. J. Med.380, 1103-1115 (2019).

75. Rini BI, Plimack ER, Stus V et al. Pembrolizumab plus axitinib versus sunitinib for advanced renal-cell carcinoma. N. Engl. J. Med.380, 1116-1127 (2019).

76. Fife KCJ, Nolasco S, Matakidou A, Welsh S, Eisen T. Metastatic renal cancer: how many patients are we treating? .Presented at: National Cancer Research Institute Cancer Conference. 4-6 November, Glasgow, UK.

77. Goebell PJ, Staehler M, Muller L et al. Changes in treatment reality and survival of patients with advanced clear cell renal cell carcinoma - analyses from the German clinical RCC-registry. Clin. Genitourin. Cancer 16(6), e1101-e1115 (2018).

78. Hirsch BR, Burke JM, Agrawal M et al. Sequential therapy in metastatic renal cell carcinoma. J. Kidney Cancer VHL 3(1), 23-35 (2016).

79. Oyinlola JO, Campbell J, Kousoulis AA. Is real world evidence influencing practice? A systematic review of CPRD research in NICE guidances. BMC Health Serv. Res. 16, 299 (2016).

- Systematic review highlights the increasing trend of the use of real-world data to influence clinical practice.

80. Skovlund E, Leufkens HGM, Smyth JF. The use of real-world data in cancer drug development. Eur. J. Cancer 101, 69-76 (2018).

81. Benchimol EI, Smeeth L, Guttmann A et al. The REporting of studies Conducted using Observational Routinely-collected health Data (RECORD) statement. PLoS Med. 12(10), e1001885 (2015).

82. von Elm E, Altman DG, Egger M et al. The Strengthening the Reporting of Observational Studies in Epidemiology (STROBE) statement: guidelines for reporting observational studies. Lancet 370(9596), 1453-1457 (2007).

83. Hemkens LG, Benchimol EI, Langan SM et al. The reporting of studies using routinely collected health data was often insufficient. $J$. Clin. Epidemiol. 79, 104-111 (2016).

84. US FDA. Cancer clinical trial eligibility criteria: minimum age for pediatric patients(2019). https://www.fda.gov/regulatory-information /search-fda-guidance-documents/cancer-clinical-trial-eligibility-criteria-minimum-age-pediatric-patients

85. US FDA. Cancer clinical trial eligibility criteria: patients with organ dysfunction or prior or concurrent malignancies (2019). https://www.fda.gov/regulatory-information/search-fda-guidance-documents/cancer-clinical-trial-eligibility-criteria-patients-organ-dys function-or-prior-or-concurrent

86. US FDA. Cancer clinical trial eligibility criteria: brain metastases (2019). https://www.fda.gov/regulatory-information/search-fda-guidance-documents/cancer-clinical-trial-eligibility-criteria-brain-metastases

87. US FDA. Cancer clinical trial eligibility criteria: patients with HIV, hepatitis B virus, or hepatitis $\mathrm{C}$ virus infections (2019). https://www.fda.gov/regulatory-information/search-fda-guidance-documents/cancer-clinical-trial-eligibility-criteriapatients-hiv-hepatitis-b-virus-or-hepatitis-c-virus 
